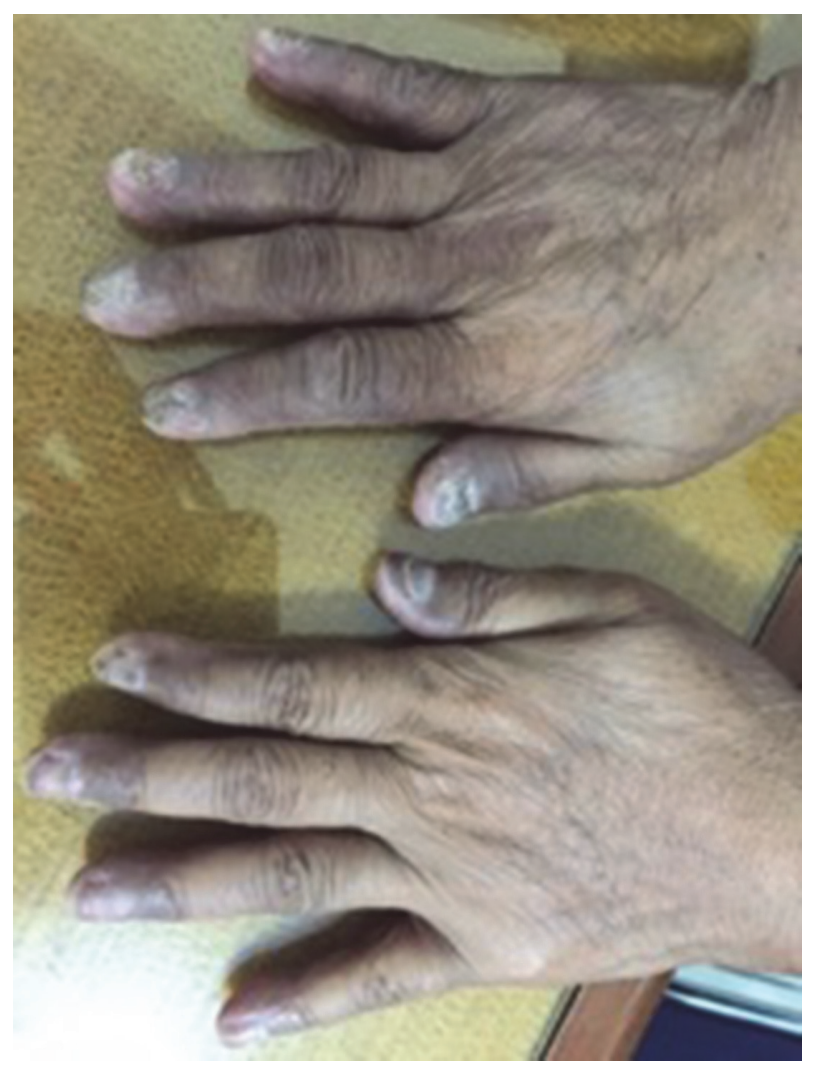

Abstract IDDF2021-ABS-0023 Figure 1

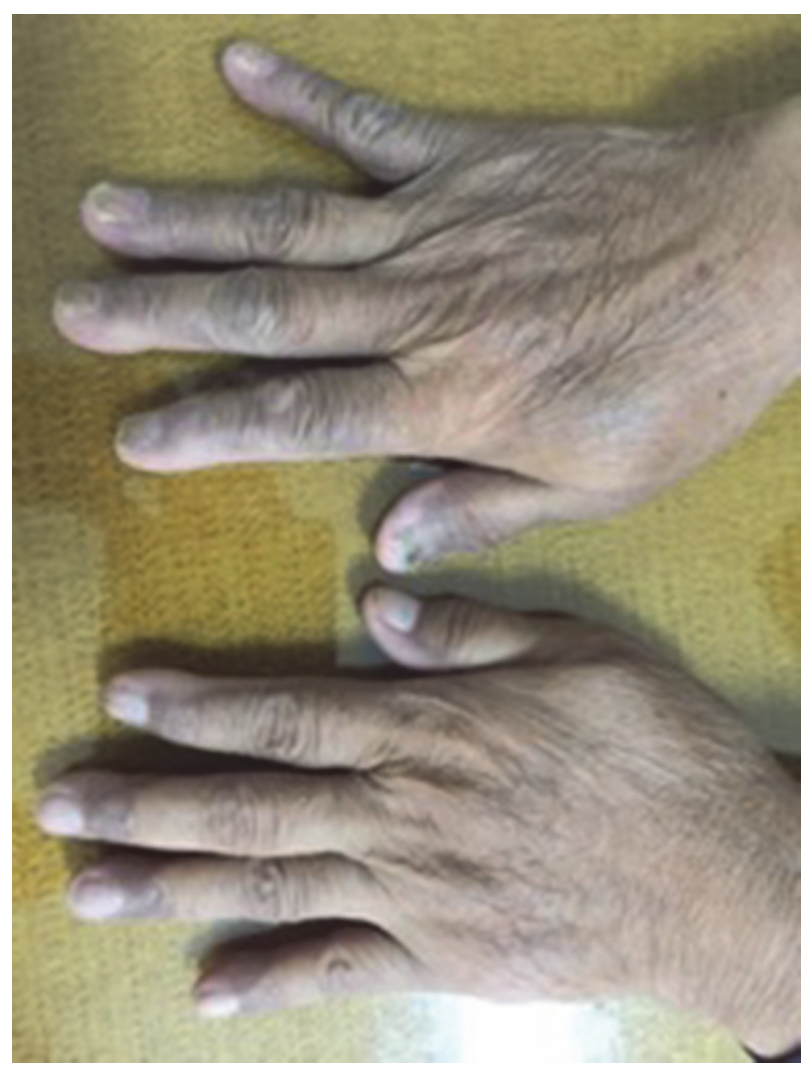

Abstract IDDF2021-ABS-0023 Figure 2
Although HFS is not a life-threatening adverse effect, it can have a great impact on the quality of life (QoL) and daily living activities of a patient. The dose interruptions and reductions are sometimes required after observation of HFS and could impact on dose intensity and clinical outcomes. As an option for the treatment of this case, we would report our single centre study in evaluating the effectiveness of MEBO for stage II and III HFS.

Methods We will evaluate the clinical sign and symptoms of hand-foot syndrome grade II and III associated with capecitabine as an adjuvant chemotherapy agent on advanced-stage colorectal cancer. All patients with HFS will be treated with topical MEBO twice daily, the clinical improvement of the symptoms will be recorded.

Results Eight cases of grade II and III hand-foot syndrome were reported during this study period, 2 patients were grade III HFS (IDDF2021-ABS-0023 Figure 1. Hand foot syndrome pre-treatment) and the others were grade II. The HFS symptoms occurred during the first 3 months after capecitabine administration. These local topical MEBO were administrated twice daily for 3 months following the chemotherapy schedule, pain reduction was achieved with no capecitabine dose interruption and reduction during the chemotherapy period. The allergic reaction was not found during and after MEBO application in this case (IDDF2021-ABS-0023 Figure 2. Hand foot syndrome post-treatment).

Conclusions Since there was no gold standard therapy, moist exposed burn ointment was an effective treatment option in managing HFS, with a good clinical response on reducing the pain without interrupting the capecitabine doses. Early recognition and multimodality treatment is the best effort in treating HFS.

\section{IDDF2021-ABS-0025 DISCRIMINATING ENDOSCOPIC FEATURES OF SESSILE SERRATED LESIONS: A SYSTEMATIC REVIEW AND META-ANALYSIS}

${ }^{1}$ Wen Shi* ${ }^{2}{ }^{2}$ Yuelun Zhang, ${ }^{3}$ Hanyue Ding, ${ }^{4}$ Feng Xie, ${ }^{1}$ Yang Chen, ${ }^{3}$ Martin CS Wong, ${ }^{1}$ Dong Wu, ${ }^{1}$ Jingnan Li. 'Department of Gastroenterology, Peking Union Medical College Hospital, Peking Union Medical College, Chinese Academy of Medical Sciences, China; ${ }^{2}$ Medical Research Center, Peking Union Medical College Hospital, Peking Union Medical College, Chinese Academy of Medical Sciences, China; ${ }^{3} J C$ School of Public Health and Primary Care, Faculty of Medicine, Chinese University of Hong Kong, China; ${ }^{4}$ Department of Health Research Methods, Evidence and Impact, McMaster University, Canada

\subsection{6/gutjnl-2021-IDDF.111}

Background Sessile serrated lesion(SSL) is notorious for its malignancy potential and the difficulty to be detected and distinguished under endoscopy. This systematic review and metaanalysis aimed to evaluate endoscopic characteristics of SSL and delineate features that inform distinction between SSA $\mathrm{P}$ and other types of lesions, including hyperplastic polyp (HP) and conventional adenoma.

Methods MEDLINE, Embase and Cochrane Library from the inception to September 9, 2020, were searched for cohort, cross-sectional or case-control studies comparing endoscopic characteristics of SSL and other polyps. The primary outcome measure was the odds of finding specific endoscopic characteristics in patients with SSL compared with other types of polys in patients undergoing colorectal cancer screening. 
Results We included 74 studies from 16 countries comprising 34,535 SSL. Compared with HP, SSL was more likely to be in the right colon (OR 5.45; 95\% CI 4.13-7.17; $\mathrm{P}<0.01$ ); larger in size $(>5 \mathrm{~mm}$ vs. $<=5 \mathrm{~mm}$ OR 5.60 ; 95\% CI $3.82-$ 8.22; $\mathrm{P}<0.01$ ); in Paris-0-II morphology (OR 2.33; 95\%CI $1.45-3.70 ; \mathrm{P}<0.01$ ); having mucus cap (OR 8.48; 95\% CI 4.86-14.8; $\mathrm{P}<0.01)$; vague margin under white light endoscopy (OR 2.71; 95\% CI 1.88-3.92; $\mathrm{P}<0.01)$; having expended crypt opening (OR 5.09; 95\% CI 1.87-13.9; $\mathrm{P}<0.01$ ), varicose microvascular vessels (OR 6.17; 95\% CI 1.57-24.3; $\mathrm{P}<0.01)$ and thick branched vessel (OR 5.17; 95\% CI 1.81-15.8; $\mathrm{P}<0.01)$ under magnified narrow band imaging and type II-O pit pattern (OR 18.56; 95\% CI 8.45-40.7; $\mathrm{P}<0.01$ ) under magnified chromoendoscopy.

Conclusions We systematically synthesised current evidence on the discriminating endoscopic features of SSL. These findings could enhance the detection of SSL in endoscopy practice.

\section{IDDF2021-ABS-0027 POST OPERATIVE PNEUMONIA AFTER ABDOMINAL SURGERY AND THE ROLE ON UNFAVORABLE OUTCOME: EVALUATION ON EMERGENCY ABDOMINAL SURGERY PATIENTS WITH COVID-19}

${ }^{1}$ Ricky Dwi Nur Tyastono*, ${ }^{2}$ Budhi Ida Bagus, ${ }^{1}$ Riza Setya Agrensa, ${ }^{1}$ Nugraha Muhammad Singgih. 'Department of Surgery, Moewardi General Hospital, Indonesia; ${ }^{2}$ Department of Surgery, Sebelas Maret University, Indonesia

\subsection{6/gutjnl-2021-IDDF.112}

Background Abdominal surgery is one of the most common procedures in a non-elective setting. Especially in the era of the COVID-19 pandemic nowadays, many kinds of abdominal surgery have been rescheduled and we must make priorities of what procedures should be done during the pandemic. Many guidelines (such as Royal College Surgery) suggested to postpone and reschedule the operative treatment during the pandemic, even in gastrointestinal cancer surgery. In this pandemic era, those guidelines could be applied in many elective settings, but not in emergency abdominal surgery procedures, we must face increasing post operative morbidity and mortality on performing surgery in confirmed COVID-19 will evaluate the post operative morbidity which leads to increased mortality of COVID-19 patients whose underwent emergency abdominal surgery during the pandemic.

Methods This study has conducted at Digestive Surgery Division at Moewardi General Hospital, Indonesia, from April 2020 until March 2021. Emergency abdominal surgery, including trauma and non-trauma cases, will be included in this study. All patients have already confirmed with positive COVID-19 pre-operatively. The procedure of this emergency surgery is laparotomy exploration with standardized level 3 PPE during surgery. The exclusion criteria of this study are to redo surgery and pediatric surgery case. The presence of post operative morbidity and in-hospital mortality will be recorded following this study.

Results From April 2020 until March 2021, we reported 39 emergency abdominal surgery cases. All of them have been done emergency exploratory laparotomy. The most common procedure is diffuse peritonitis due to peptic ulcer perforation (reported in 21 cases), other procedures are complicated appendicitis in 9 cases, two cases of blunt abdominal trauma, large bowel obstruction was found in 4 cases and 3 cases of incarcerated groin hernia. Thirty-one patients survived following the emergency abdominal surgery and 8 patients did not survive with post operative pneumonia and uncontrolled sepsis in the ICU.

Conclusions Post operative pneumonia is the important factor which has an impact on unfavorable clinical outcome during emergency abdominal surgery procedures on confirmed COVID-19 positive patients. These high-risk procedures need the perioperative multidiscipline team to gain a better outcome in this current pandemic.

\section{IDDF2021-ABS-0035 OPIUM ADDICTION INDUCED GASTRIC OUTLET OBSTRUCTION- AN UNUSUAL ENTITY}

Sudhir Maharshi*, Shyam Sunder Sharma. SMS Medical College and Hospitals, Jaipur, India

\subsection{6/gutjnl-2021-IDDF.113}

Background There is a paucity of data in the literature on opium addiction causing gastric outlet obstruction. The aim was to study opium addiction causing gastric outlet obstruction and to assess the role of endotherapy.

Methods Prospective study was conducted at SMS Hospital, Jaipur, between January 2017 to December 2020. All patients with a clinical diagnosis of gastric outlet obstruction with a history of opium addiction were consecutively enrolled. Data relating to demography, laboratory parameters, radiological imaging, histology and outcome of endotherapy were analyzed.

Results Sixty patients (age $38.3 \pm 11.2$ years, BMI $17.6 \pm 2.9 \mathrm{~kg}$ / $\mathrm{m}^{2}, 57$ males) were studied. Site of involvement was pylorus with junction of first and second part of duodenum in 24 $(40 \%)$, junction of first and second part of duodenum in 16 (26.7\%), pylorus with distal second part or third part of duodenum in $11(18.3 \%)$ as shown in figure $1 \& 2$ and pylorus, junction of first and second part of duodenum along with distal second part of duodenum or third part seen in $9(15 \%)$ patients (IDDF2021-ABS-0035 Figure 1. Endoscopic image showing ulcerated stricture at pylorus, IDDF2021-ABS-0035 Figure 2. Endoscopic image showing stricture in distal second

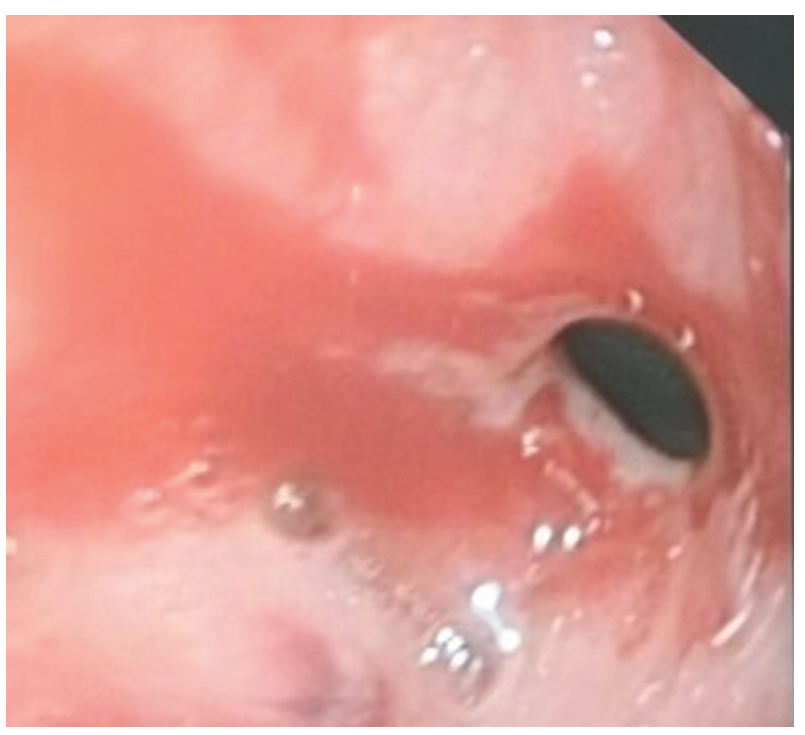

Abstract IDDF2021-ABS-0035 Figure 1 